\title{
Paradigm shift of drug information centers during the COVID-19 pandemic
}

\author{
Asmita Priyadarshini Khatiwada ${ }^{1}$ (D) Sujyoti Shakya ${ }^{1,2} \cdot$ Sunil Shrestha ${ }^{1,3}$ (I)
}

Published online: 20 July 2020

(c) Springer Nature Switzerland AG 2020

\begin{abstract}
The novel coronavirus disease 2019 (COVID-19) pandemic is a major global threat affecting millions of lives throughout the world physically and psychologically. With the asymptomatic presentation of COVID-19 in many patients and the similarity of its symptoms with the common cold and influenza, the need for accurate information on the disease is very important for its identification and proper management. Accurate information on the disease, its prevention and treatment can be disseminated through drug information centers (DICs). DICs are usually staffed by pharmacists and/or clinical pharmacists/pharmacologists. DICs are a reliable source of current and unbiased information on COVID-19 and its associated complications, including management options for healthcare professionals and the public. In addition to health and drug information, pharmacists working in the DICs can be involved in the management of the patients' health by providing information on home care and safety, medication management of patients with chronic comorbid illnesses, and psychological advice. This article explores the possible additional roles DICs can play, besides providing drug information within the hospital or in the community.
\end{abstract}

\section{Introduction}

With the initiation of the outbreak in Wuhan, China in December 2019, the pandemic of novel coronavirus disease 2019 (COVID-19) has become a worldwide threat. Psychologically and/or physically, COVID-19 has deep effects on people throughout the globe. COVID-19 is caused by severe acute respiratory syndrome coronavirus 2 (SARS-CoV-2), and people may present as asymptomatic or with symptoms such as fever, cough, tiredness, headache, and difficulty breathing [1]. As the clinical presentation of COVID-19

All authors have an equal contribution.

Asmita Priyadarshini Khatiwada

asmitapriyadarshinikhatiwada@gmail.com

Sujyoti Shakya

ssujyoti@gmail.com

Sunil Shrestha

sunilcresta@gmail.com

1 Department of Pharmaceutical and Health Service Research, Nepal Health Research and Innovation Foundation, Lalitpur, Nepal

2 Department of Pharmacy, Nobel College, Kathmandu, Nepal

3 Department of Pharmacy, Nepal Cancer Hospital and Research Center Pvt. Ltd, Lalitpur, Nepal symptoms is similar to that of the common cold and influenza, complete and reliable information on the disease, testing methods, and prevention is of utmost importance for the public and healthcare professionals/providers (HCPs) [2]. Such information can be gathered, validated and provided to the public by drug information centers (DICs).

DICs are usually found within hospitals or in the community setting, are staffed by pharmacists, and are accountable for the communication of recent, unbiased, critically reviewed information on medications [3]. Not all DICs serve the public; however, during the current pandemic, DICs can serve as information hubs for providing current and reliable information to HCPs and patients/consumers regarding COVID-19 and its related complications, symptom management, interactions with co-morbid conditions, and drug-drug interactions (DDIs).

However, providing drug information and other DIC services to the public may not be easy in many lower-income countries (LICs), as HCPs are not evenly distributed across geographical regions [4], and resources and manpower are limited [5, 6]. In LICs, managing COVID-19 seems difficult, as they lack resources to hire or train new HCPs, leaving them with the alternative of maximizing the use of existing human resources, including pharmacists. Existing pharmacist manpower could serve the public by providing 
information on COVID-19 and its transmission, prevention, and management within hospitals and communities.

\section{Need for awareness about COVID-19}

With the global spread of COVID-19, providing accurate information about the disease is an issue for everyone, including HCPs, patients, and the public. Continuing medication education programs and training on the effective management of patients with COVID-19 can be arranged for HCPs. Additionally, training focused on patients presenting with COVID-19 symptoms along with various comorbid conditions may be highly effective. To prevent the complications arising from COVID-19 and existing comorbidities, appropriate knowledge of management strategies is crucial for patient safety. As the infection transfers from human to human through respiratory droplets and contacts [7], the public needs to be aware of COVID-19, with current information on the spread and prevention of the disease. To deal with unforeseen situations such as COVID-19, in addition to government preparedness, health literacy among the general population and HCPs regarding the disease is important [8].

\section{Pharmacist involvement during pandemics}

During outbreaks or pandemics, pharmacists are known to effectively work as frontline HCPs [9-12]. Pharmacists played a crucial role in direct patient care, medication information, and proper drug distribution with proactive communication among themselves and with other HCPs during the severe acute respiratory syndrome (SARS) pandemic. Pharmacists were among the major stakeholders responsible for developing guidelines for the administration of oral and intravenous antivirals, updating hospital formularies, providing information on investigational drugs, and providing information on dosage adjustments in hepatic and renal failure patients [11]. During an outbreak of H1N1 influenza, pharmacists were also involved in improving patient awareness. They provided information about the disease and preventive measures to the patients through community pharmacies, and acted as immunization providers in collaboration with public health associates $[10,13]$.

The pharmacists' role during the Ebola virus epidemic in Africa was minimal, with no additional activities being undertaken. However, new roles including immunization, contraception, public awareness, identifying infected patients and redirecting them to hospitals and isolation centers, logistics, supplies and clinical management, and being an information hub for patients and HCPs regarding the disease, transmission, preventive measures, management approaches, and investigational medications were identified $[14,15]$.

Even though the various services provided by the pharmacists during different pandemics were not directly through DICs, pharmacists were actively involved in the dissemination of information on the disease and investigational World Health Organization (WHO)-approved/non-approved medications to the patients, HCPs and the public.

\section{Extended role of DICs in the COVID-19 pandemic}

DICs can serve as a focal point for the dissemination of unbiased information to the patients/public regarding COVID-19 and its treatment and management [16]. This will upgrade the role of DICs in patient care by acting as a 'Disease Information Center', in addition to providing drug information. Detailed information on COVID-19 and its effects in patients with various health conditions, such as cardiovascular disease (CVD), diabetes mellitus, neurological issues, and respiratory illnesses, can be disseminated to HCPs and patients/ public via DICs, thereby promoting the role of pharmacists in patient management. The following sections cover the broad role of DICs as Disease Information Centers.

\section{DICs' role in chronic illness and comorbidities}

Patients with a chronic illness, such as diabetes, CVD, and kidney disease, usually have many questions regarding medications and lifestyle modifications [17]. Such questions may be more frequent during the COVID-19 outbreak, as patients may consider themselves to be at high risk for the infection. The management of chronic conditions is challenging because of their various complications, multiple and complicated drug regimens, and fluctuating disease states and patterns [18]. Polypharmacy is associated with comorbidities and negative clinical outcomes [19]. Epidemiological data show that older patients with several comorbidities are more susceptible to COVID-19-related health issues than those without [20]. Proper communication regarding the prevention and spread of infection in this vulnerable patient group can be done by DIC pharmacists in collaboration with other public HCPs, which may allow frontline HCPs more time to care for their infected patients.

With the help of their documentation, DICs can contact and follow patients with chronic conditions to whom DIC services had been provided earlier, which may improve their health outcomes. In many LICs, there is a lack of e-prescribing software, making patient follow-up difficult. Where available, however, pharmacy databases used mainly to record sales and purchases of medicines, are valuable in identifying patients with chronic illnesses, as their 
prescribed drugs suggest their comorbidities [21]. This may represent an additional role for DICs in hospitals and communities, but relies on DICs having staff available to perform such duties, the preparation and utilization of standard operating procedures, and the ability to access patient information in both the hospital and community settings. DICs can also greatly aid HCPs by providing current, complete, and accurate information on drug therapy and management approaches for treating COVID-19 patients with various comorbid conditions based on the scientific literature, and national and international guidelines.

\section{DICs' role in drug interactions and adverse effects}

Information regarding adverse drug reactions (ADRs) and DDIs is often sought by patients, especially those with diabetes, CVD, or respiratory diseases [22]. Although online information can be easy to access, the reliability and validity of such information is often questionable. Patients with multiple comorbidities deal with psychological distress, and are often poorly compliant concerning medication administration and lifestyle modifications, leading to reduced quality of life [23]. This seems to be more likely in the absence of appropriate knowledge on the disease conditions, medications, DDIs, drug-food interactions, drug-disease interactions, and ADRs [24-26]. This highlights the important role that DICs can play as a disease information center, particularly in this health crisis, and may contribute to better patient outcomes in collaboration with other HCPs [27].

If direct face-to-face communication of information is not possible, remote communication via teleconferencing can be used [28]. Monitoring of ADRs may be difficult during COVID-19 lockdowns. Proactive communication is very important for proper assessment of ADRs, with pharmacists able to be actively involved in assessing, monitoring, and detecting ADRs. This may also be a role for DICs and, to ensure smooth functioning of their diverse functions, requires DICs to be adequately staffed and resourced. Patients should be advised to be alert to the development of unwanted ADRs, and to provide the DIC with the details via telephone, email, or other means.

\section{DICs' role in home care and safety}

According to the WHO, staying home and maintaining social distancing can help to prevent the spread of COVID19 , as no vaccine or specific treatment is currently available [29]. DICs can play an important role in providing information regarding home care and safety during the COVID-19 pandemic.

DICs can encourage HCPs to evaluate the basic recommendations given by reliable organizations, such as the WHO, Mayo Health Centres, US Centers for Disease
Control and Prevention, and others, for care at home during this pandemic. Recommendations may include evaluating whether the patient is stable enough to receive care at home, and the availability of caregivers at home and the isolation area; and suggestions on maintaining required distances with infected individuals, sanitization, and cleaning. DICs could also provide information on the accessibility of food and other necessities, including personal protective equipment (PPE; at the very least, gloves and facemasks) [30, 31]. HCPs should educate patients/public on measures such as self-isolation; not sharing personal items; avoiding touching the eyes, nose, and mouth; washing hands frequently for at least $20 \mathrm{~s}$ or using sanitizers; cleaning and sterilizing surfaces; and ignoring unwanted visitors while providing home care for mildly symptomatic individuals [32].

Furthermore, DICs should also be involved in the evaluation of the presence of any vulnerable individuals who are at increased risk of COVID-19 (e.g. those aged $>65$ years, young children, pregnant women, immunocompromised individuals, and those with chronic heart, lung, or kidney conditions), and provide information on the measures to be taken if any unusual symptoms or symptoms of severe COVID-19 develop [30, 31].

\section{DICs' role in communication via social media}

The public often use social media when seeking information and solutions for health issues. Social media can also be an effective aid in providing healthcare services to patients (e.g., online consultation with pharmacists regarding medication-related issues), as well as for sharing and acquiring information among HCPs [33].

DIC pharmacists can use the official social media pages of DICs for healthcare communication, sharing health and medicine information, locating medicines for patients, and providing medicines in case of shortages. This will limit consumer visits to HCPs during the pandemic. For example, when a patient approaches the DIC for a medication, they can contact the pharmacies in their network regarding drug availability, then provide the patient with the details. Such communication can be done through social media sites such as WhatsApp, Viber, Facebook, Twitter, etc. Information on the availability of medications could also be gathered and, with permission from the pharmacies, provided on the social media pages of the DIC, which will be of great help for patients. This will help develop public trust regarding the other services provided by the DIC and limit unnecessary travel of people outside the home, preventing them from being exposed to the virus.

New and updated information on medications approved for COVID-19 treatment or prevention can be posted on social media sites of DICs. 


\section{DICs' role in informing about current COVID-19 treatment}

Initially, hydroxychloroquine, a drug used to treat malaria and inflammation related to autoimmune disorders, was authorized for use in COVID-19 by the US Food and Drug Administration (FDA) through an Emergency Use Authorization (EUA) [34, 35]. However, cases of hospitalization and death due to self-medication of this drug indicate that dissemination of correct information regarding its use is necessary [36, 37]. Given data from ongoing trials and emerging studies suggesting that hydroxychloroquine and chloroquine do not help to decrease the mortality or speed the recovery of COVID-19 patients, and the potential for life-threatening heart rhythm problems, the FDA withdrew the EUA for their use in COVID-19 [38]. Nevertheless, trials exploring the use of hydroxychloroquine and chloroquine in the prevention of COVID-19 are to be restarted, as there are issues regarding the results of the study depicting the harmful effect of these drugs, based on which the WHO had earlier suspended the use of these drugs in COVID-19 [39].

An EUA was issued also for remdesivir, an investigational antiviral agent in the treatment of hospitalized COVID-19 patients. However, it is not yet approved by the FDA as the drug is still undergoing clinical trials [40, 41].

Recently, dexamethasone, a well-known corticosteroid, was shown to decrease COVID-19 mortality in patients with serious respiratory problems, but do not have any beneficial effect in patients with mild symptoms, according to preliminary results of the RECOVERY trial at Oxford University $[42,43]$.

There are many ongoing clinical trials in the quest for treatments for COVID-19. Among the plethora of information and frequently changing notions regarding treatment, appropriate information must be delivered to patients and HCPs. DICs can play a significant role in providing information to HCPs and the public regarding the use and effects of new medications being authorized and other medications being investigated for COVID-19.

\section{Patients and consumers}

Patients currently taking hydroxychloroquine/chloroquine to treat approved indications, such as malaria and autoimmune conditions, should continue to take the drug, and be advised not to stop taking their medicine without consulting a HCP [44]. DICs should inform new users not to consume these medicines without knowledge of their HCPs, due to the risk of severe poisoning and death with their non-prescribed use [44]. They should be counseled to seek immediate medical attention if they experience irregular heartbeat, dizziness, or fainting while receiving hydroxychloroquine/chloroquine, and to store the drugs beyond the reach of children. The same advice also pertains to dexamethasone in the context of regular use, new users and approaching HCPs for help if ADRs occur.

\section{Health professionals}

DICs should ensure HCPs are aware of the current position of investigational drugs hydroxychloroquine/chloroquine, remdesivir, and dexamethasone (i.e., they are not yet approved to prevent or treat COVID-19, but their use should be continued in patients taking them for other conditions). As some individuals may still take hydroxychloroquine/chloroquine for COVID-19, HCPs should be aware of the potential ADRs associated with these drugs, such as QT prolongation (increased risk in patients with renal insufficiency or failure), increased insulin levels and insulin action (increasing the risk of severe hypoglycemia), hemolysis in patients with glucose-6-phosphate dehydrogenase deficiency, and DDIs with drugs that cause QT prolongation (e.g., azithromycin) [45]. DICs should alert HCPs regarding the possible ADRs of dexamethasone (e.g., hyperglycemia, secondary infections, hypertension, fluid retention, and exacerbation of systemic fungal infections) [46], as well as its appropriate dosage and patient profile. HCPs should ensure that patients receiving the drug do not have any existing conditions that might be exaggerated by dexamethasone.

\section{DICs' role in providing information on symptom management}

Based on the scientific evidence and expert opinion, DICs can provide up-to-date information on the management of COVID-19. People should be informed that immediate hospitalization is not necessary in cases of mild clinical presentation (lack of viral pneumonia and hypoxia), and that the initial symptoms can be easily managed at home [47]. DICs should encourage patients to seek immediate medical attention if they experience breathing problems, persistent chest pain and chest tightness, dizziness or inability to wake up, or their lips or face turn blue [32].

Some patients with COVID-19 need to be hospitalized for serious illness and associated comorbid conditions. Inpatient management revolves around adjuvant management of common complications of severe COVID-19, such as pneumonia, hypoxemic respiratory failure/acute respiratory distress syndrome (ARDS), sepsis and septic shock, cardiomyopathy and arrhythmia, acute kidney injury, and chronic hospital complications, secondary bacteriostoma, thromboembolism, and serious disease polyneuropathy/ myopathy [48-51].

Patients with severe acute respiratory infections (SARI) and respiratory problems, hypoxemia, or shock require immediate complementary oxygen therapy. Patients 
presenting with severe COVID-19 symptoms should be closely monitored for clinical decline (e.g., rapid progressive respiratory failure and sepsis) and immediate treatment with adjuvant interventions should be provided. Empiric antimicrobial therapy should be given as soon as possible to treat SARI. Advanced oxygen/ventilator support should be considered for severe hypoxemic respiratory failure when standard oxygen therapy fails in patients with respiratory problems. Corticosteroids are not routinely recommended for viral pneumonia or ARDS, and should be avoided unless indicated for another reason (e.g., chronic obstructive pulmonary disease exacerbations, refractory septic shock) [32].

\section{Pharmacists' role as psychological support}

With the global chaos of the COVID-19 pandemic, mental health is an issue of concern. Pharmacists can play a significant role in managing the psychological trauma, insecurity, and depression in individuals during this threatening period. Although a psychologist is a more suitable HCP, pharmacists can also provide psychological support by communicating with patients, giving information on mental health issues along with the contact details of trained counselors, if needed [52, 53]. Pharmacists can counsel patients in the one-to-one session via telephone or social media, without the need for a physical visit. There is a scope for DICs to liaise with different stakeholders to develop online support groups especially focused on patients with chronic illnesses, as group discussions may help them manage their conditions and prevent any mental effects amid quarantine and lockdowns.

\section{Take home messages}

- During the global threat of COVID-19, all HCPs have significant and crucial roles to play.

- DICs, staffed with pharmacists, can be involved in achieving better health outcomes for patients/consumers by enhancing their role across the disease management process.

- To enhance their role, DICs can provide information on COVID-19, including management approaches, psychological advice, home care and safety, DDIs and ADRs, and medical management of chronic comorbid illnesses, in addition to their usual role in providing drug information.

- Further, the liaison of DICs with the different national health authorities and organizations might ensure the better utilization of DIC services by the people of the country.
Acknowledgements The authors would like to acknowledge Dr. Saval Khanal, Research Fellow in health economics and behavioral sciences at Warwick University, UK, for the suggestions and modifications during revision of the manuscript.

Author contributions AP Khatiwada developed the study concept, conducted the literature review, and wrote the first draft of the manuscript. S Shakya made substantial changes to the study concept, conducted a literature review, and provided support during the writing of the first draft. S Shrestha provided feedback on the study concept, and critically revised and reviewed the manuscript. All authors approved the final version of the manuscript.

\section{Declarations}

Funding This research did not receive any funding from any agency in the public, commercial, or not-for-profit sectors

Conflict of interest The authors declare that they have no conflicts of interest.

\section{References}

1. World Health Organization (WHO). Coronavirus. 2020. https:// www.who.int/health-topics/coronavirus\#tab=tab_3. Accessed 15 May 2020.

2. Kakodkar P, Kaka N, Baig M. A comprehensive literature review on the clinical presentation, and management of the pandemic coronavirus disease 2019 (COVID-19). Cureus. 2020;12(4):e7560.

3. Shrestha S, Khatiwada AP, Gyawali S, et al. Overview, challenges and future prospects of drug information services in Nepal: a reflective commentary. J Multidiscip Healthc. 2020;13:287-95.

4. Global Pharmacy Workforce and Migration Report. Workforce description. 2009. https://www.fip.org/files/fip/HR/final\%2520r eport/Part1.pdf. Accessed 15 May 2020.

5. Scheffler RM, Bruckner T, Spetz J. The labour market for human resources for health in low- and middle-income countries. Human Resources for Health Observer - Issue No. 11. 2012; pp. 1-20.

6. Bangdiwala SI, Fonn S, Okoye O, et al. Workforce resources for health in developing countries. Public Health Rev. 2010;32(1):296-318.

7. World Health Organization (WHO). Modes of transmission of virus causing COVID-19: implications for IPC precaution recommendations. 2020. https://www.who.int/news-room/commentari es/detail/modes-of-transmission-of-virus-causing-covid-19-impli cations-for-ipc-precaution-recommendations. Accessed 16 May 2020.

8. Paakkari L, Okan O. COVID-19: health literacy is an underestimated problem. Lancet Public Health. 2020;5(5):e249-e250.

9. Role of hospital pharmacists during pandemic flu updated. Hosp Pharmacist. 2007;14:361.

10. Association of State and Territorial Health Officials (ASTHO). Public health and pharmacy collaboration in an influenza pandemic: summary of findings from an exploratory interview project. Arlington (VA): ASTHO; 2020.

11. Chin TWF, Chant C, Tanzini R, et al. Severe acute respiratory syndrome (SARS): the pharmacist's role. Pharmacotherapy. 2004;24(6):705-12.

12. Guarascio AJ, Faust AC, Sheperd L, et al. Ebola virus disease. Ann Pharmacother. 2014;49(2):247-9.

13. Miller S, Patel N, Vadala T, et al. Defining the pharmacist role in the pandemic outbreak of novel H1N1 influenza. J Am Pharm Assoc. 2012;52(6):763-7. 
14. James PB, Jamshed SQ, Patel I. Ebola virus disease: how can African pharmacists respond to future outbreaks? Asian Pac J Trop Dis. 2016;6(5):337-8.

15. Tariq A. The role of pharmacists in the EVD outbreak. RhoChi Post. 2015;4(7):2-3.

16. Umashankar MS, Lakshmi KS, Kumar AB, et al. Review on benefits of drug information center services: a new transpiring practice to health care professionals in hospitals. J Chem Pharm Res. 2017;9(5):28-38.

17. Tefera YG, Gebresillassie BM, Ayele AA, et al. The characteristics of drug information inquiries in an Ethiopian university hospital: a two-year observational study. Sci Rep. 2019;9:13835.

18. Mahajan N. Good pharmacy practice in chronic disease management. Indian J Pharm Pract. 2009;1(2):18-20.

19. Huber CA, Szucs T, Rapold R, et al. Identifying patients with chronic conditions using pharmacy data in Switzerland: an updated mapping approach to the classification of medications. BMC Public Health. 2013;13:1030.

20. Sanyaolu A, Okorie C, Marinkovic A, et al. Comorbidity and its impact on patients with COVID-19. SN Compr Clin Med. 2020. https://doi.org/10.1007/s42399-020-00363-4.

21. Castle SC, Uyemura K, Rafi A, et al. Comorbidity is a better predictor of impaired immunity than chronological age in older adults. J Am Geriatr Soc. 2005;53(9):1565-9.

22. Kusch MKP, Haefeli WE, Hanna M, et al. How to meet patients' individual needs for drug information: a scoping review. Patient Prefer Adherence. 2018;12:2339-555.

23. Ekici M, Ekici A. The correlations between the presence of comorbidities, psychological distress and health-related quality of life. Handbook of disease burdens and quality of life measures. New York: Springer; 2010. p. 3475-88.

24. Polanska BJ, Uchmanowicz I, Dudek K, et al. Relationship between patients' knowledge and medication adherence among patients with hypertension. Patient Prefer Adherence. 2016;10:2437-47.

25. Mekonnen GB, Gelayee DA. Low medication knowledge and adherence to oral chronic medications among patients attending community pharmacies: a cross-sectional study in a low income country. Biomed Res Int. 2020;2020:4392058.

26. Salama AA, Yasin AEA, Elbarbary W. Medication knowledge as a determinant of medication adherence in geriatric patients, Serse Elian city, Menoufia Governorate, Egypt. Menoufia Med J. 2017;30(1):63-8.

27. Ashenef A, Reshid E, Yilma Z, et al. Assessment of the use and status of new drug information centers in a developing country, Ethiopia: the case of public university hospital drug information centers. Biomed Res Int. 2018;2018:3840976.

28. Bukhari N, Rasheed H, Nayyer B, et al. Pharmacists at the frontline beating the COVID-19 pandemic. J Pharm Policy Pract. 2020;13:8.

29. World Health Organisation (WHO). Coronavirus disease (COVID19) advice for the public. 2020. https://www.who.int/emergencie s/diseases/novel-coronavirus-2019/advice-for-public. Accessed May 172020.

30. Centers for Disease Control and Prevention. Coronavirus disease 2019: caring for patients at home. https://www.cdc.gov/coronaviru s/2019-ncov/hcp/guidance-home-care.html. Accessed 16 May 2020.

31. American Red Cross. COVID-19: safety tips for you. https://www. redcross.org/about-us/news-and-events/news/2020/coronaviru s-safety-and-readiness-tips-for-you.html. Accessed 17 May 2020.

32. World Health Organization. Severe acute respiratory infections treatment centre: practical manual to set up and manage a SARI treatment centre and SARI screening facility in health care facilities. Geneva: World Health Organization; 2020
33. Benetoli A, Chen TF, Schaefer M, et al. Professional use of social media by pharmacists: a qualitative study. J Med Internet Res. 2016;18(9):e258.

34. Schrezenmeier E, Dörner T. Mechanisms of action of hydroxychloroquine and chloroquine: implications for rheumatology. Nat Rev Rheumatol. 2020;16(3):155-66.

35. US FDA. Hydroxychloroquine or chloroquine for COVID-19: drug safety communication-FDA cautions against use outside of the hospital setting or a clinical trial due to risk of heart rhythm problems. https://www.fda.gov/safety/medical-product-safet y-information/hydroxychloroquine-or-chloroquine-covid-19-drugsafety-communication-fda-cautions-against-use. Accessed 28 June 2020.

36. Smith C. The CDC just changed key info about hydroxychloroquine on its coronavirus site. https://bgr.com/2020/04/09/ coronavirus-usa-cdc-fixes-hydroxychloroquine-covid-19-info/. Accessed 17 May 2020.

37. Park A. President Trump called hydroxychloroquine a 'game changer,' but experts warn against self-medicating with the drug. Here's what you need to know. https://time.com/5808894/hydro xychloroquine-coronavirus/. Accessed 18 May 2020.

38. US FDA. Memorandum explaining basis for revocation of emergency use authorization for emergency use of chloroquine phosphate and hydroxychloroquine sulfate. https://www.fda.gov/media /138945/download. Accessed 28 June 2020.

39. BBC News. Coronavirus: hydroxychloroquine trial to restart. https ://www.bbc.com/news/health-53233070. Accessed 2 July 2020.

40. US FDA. Letter of authorisation for emergency use of remdesivir for treatment of COVID-19. 2020. https://www.fda.gov/media /137564/download. Accessed 29 June 2020.

41. US FDA. Coronavirus (COVID-19) update: FDA issues emergency use authorisation for potential COVID-19 treatment. https ://www.fda.gov/news-events/press-announcements/coronaviru s-covid-19-update-fda-issues-emergency-use-authorization-poten tial-covid-19-treatment. Accessed 29 June 2020.

42. Roberts M. Coronavirus: Dexamethasone proves first life-saving drug. BBC news online. 2020. https://www.bbc.com/news/healt h-53061281. Accessed 29 June 2020.

43. World Health Organisation (WHO). WHO welcomes preliminary results about dexamethasone use in treating critically ill COVID19 patients. 2020. https://www.who.int/news-room/detail/16-062020-who-welcomes-preliminary-results-about-dexamethasoneuse-in-treating-critically-ill-covid-19-patients. Accessed 29 June 2020.

44. US FDA. Hydroxychloroquine or chloroquine for COVID-19: drug safety communication - FDA cautions against use outside of the hospital setting or a clinical trial due to risk of heart rhythm problems. https://www.fda.gov/safety/medical-product-safet y-information/hydroxychloroquine-or-chloroquine-covid-19-drugsafety-communication-fda-cautions-against-use. Accessed 19 May 2020.

45. US FDA. MedWatch: the FDA safety information and adverse event reporting program. 2020. https://www.fda.gov/safety/medwa tch-fda-safety-information-and-adverse-event-reporting-program. Accessed 23 May 2020.

46. Decadron (dexamethasone tablets, USP). Silver Spring: US Food and Drug Administration; 2018.

47. Huang C, Wang Y, Li X, et al. Clinical features of patients infected with 2019 novel coronavirus in Wuhan. China Lancet. 2020;395(10223):497-506.

48. Zhou F, Yu T, Du R, et al. Clinical course and risk factors for mortality of adult inpatients with COVID-19 in Wuhan, China: a retrospective cohort study. Lancet. 2020;395(10229):1054-62.

49. Yang X, Yu Y, Xu J, et al. Clinical course and outcomes of critically ill patients with SARS-CoV-2 pneumonia in Wuhan, China: 
a single-centered, retrospective, observational study. Lancet Respir Med. 2020;8(4):475-81.

50. Chen N, Zhou M, Dong X, et al. Epidemiological and clinical characteristics of 99 cases of 2019 novel coronavirus pneumonia in Wuhan, China: a descriptive study. Lancet. 2020;395(10223):507-13.

51. Wang D, Hu B, Hu C, et al. Clinical characteristics of 138 hospitalized patients with 2019 novel coronavirus-infected pneumonia in Wuhan, China. JAMA. 2020;323(11):1061-9.
52. Zhenga S, Yanga L, Zhoua P, et al. Recommendations and guidance for providing pharmaceutical care services during COVID19 pandemic: a China perspective. Res Soc Adm Pharm. 2020. https://doi.org/10.1016/j.sapharm.2020.03.012.

53. Beyond prescriptions: what patients need during the COVID-19 pandemic. US Pharm. 2020. Epub 22 May 2020. 